\title{
Variability in the extreme helium star LSS $5121^{\star}$
}

\author{
V. M. Woolf ${ }^{1}$, R. Aznar Cuadrado ${ }^{1}$, G. Pandey ${ }^{2}$, and C. S. Jeffery ${ }^{1}$ \\ 1 Armagh Observatory, College Hill, Armagh BT61 9DG, Northern Ireland \\ e-mail: rea@star.arm.ac.uk; csj@star.arm.ac.uk \\ 2 Department of Astronomy, University of Texas, Austin, TX 78712, USA \\ e-mail: pandey@astro.as.utexas.edu
}

Received 6 February 2001 / Accepted 14 March 2001

\begin{abstract}
We report a photometric and spectroscopic study of the hot extreme helium star LSS 5121. We found photometric variability, but no period was evident in its periodogram. This is consistent with the previous proposal, based on spectral line variations, that LSS 5121 is a non-radial pulsator similar to other hot extreme helium stars.
\end{abstract}

Key words. stars: chemically peculiar - stars: oscillations - stars: variables - stars: individual: LSS 5121

\section{Introduction}

Extreme helium stars (EHes) are luminous blue stars with spectra that indicate surfaces composed almost entirely of helium, with traces of carbon and nitrogen and very little (usually $\ll 1$ per cent) hydrogen. Their temperatures fall in the range $8000 \mathrm{~K} \leq T \leq 32000 \mathrm{~K}$. The EHes are of interest because, among other things, some of them pulsate or are otherwise variable, they have very unusual chemical compositions, and they are quickly evolving, with the radii and temperature changes of some being measurable over a period of a decade or less (Jeffery et al. 2001). Although their evolutionary history is still not completely certain, it is evident that they are post AGB stars evolving to become white dwarfs. EHes evolution may be linked with other hydrogen deficient star classes such as RCrB stars and helium subdwarf B stars (He sdBs). It may be that a sizable fraction of stars go through an EHe phase.

Pulsations in EHes are excited through iron group (Zbump) opacities (Saio 1993). The pulsations in some EHes allow accurate estimates to be made of their physical properties, including their mass and radii (Lynas-Gray et al. 1984, 1987; Woolf \& Jeffery 2000). The study of pulsations in stars with atmospheres not dominated by hydrogen (e.g. EHes and He sdBs) provides additional constraints on pulsation theory. Fewer than 30 EHes are known (Jeffery et al. 1996) and few of those have been studied in any detail. The

Send offprint requests to: V. M. Woolf,

e-mail: vmw@star.arm.ac.uk

* Based on observations made at the University of Texas McDonald Observatory, Fort Davis, Texas and the Jacobus Kapteyn Telescope operated on the island of La Palma by the Isaac Newton Group in the Spanish Observatorio del Roque de los Muchachos of the Instituto de Astrofisica de Canarias. physical properties and the variability of additional EHes must be determined if a statistical study of the group is to have meaning.

LSS 5121 is a hot extreme helium star. The temperature of LSS 5121 has been estimated as $28300 \mathrm{~K}$ (Heber et al. 1986) using $U B V$ color measurements and $29772 \mathrm{~K}$ (Jeffery et al. 2001) by comparing optical photometry and IUE ultraviolet spectra with the calculated theoretical flux distributions. Earlier spectroscopic observations by Lawson \& Kilkenny (1998) demonstrated that its absorption lines vary in strength on a timescale of a few days. The line variations are similar to those observed in the $(T \approx 20 \mathrm{kK})$ EHe $\mathrm{BD}-9^{\circ} 4395$, a nonradial pulsator (Jeffery \& Heber 1992). The spectrum of LSS 5121 is very similar to that of the $(T \approx 29 \mathrm{kK}) \mathrm{EHe}$ HD 160641 (Heber et al. 1986), which has been found to pulsate non-radially with several pulsation periods which range between 8.4 hours and 1.77 days (Lynas-Gray et al. 1987). The spectral similarity indicates similar temperatures, gravities, and chemical compositions. The gravity of LSS 5121 has not been determined, but it is clear from its spectrum that its lies between those of the EHes

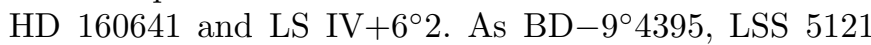
and HD 160641 all lie near or above the Z-bump instability boundary (Saio \& Jeffery 1999) it would be reasonable to suppose that LSS 5121 may also pulsate. Given the similarity of the physical properties of LSS 5121 and HD 160641, one might also expect LSS 5121 to pulsate non-radially. EHes with physical properties like those of LSS 5121 tend to vary on timescales of hours to days, rather than minutes or months. Our observations were designed to detect variability on the expected timescales.

LSS 5121 was too $\operatorname{dim}, m_{V}=13.3$, for Lawson \& Kilkenny (1998) to test for photometric variations with the 
Table 1. Observations

\begin{tabular}{ll}
\hline Telescope & HJD -2451000 \\
\hline JKT & $677.5626-677.7374$ \\
JKT & $678.5567-677.7344$ \\
McD 0.8-m & $706.8056-706.9503$ \\
McD 0.8-m & $707.9320-707.9538$ \\
McD 0.8-m & $709.7630-709.9532$ \\
McD 0.8-m & $710.7639-710.9643$ \\
McD 0.8-m & $711.7452-711.9635$ \\
McD 0.8-m & $717.7015-717.9539$ \\
McD 2.1-m & $709.7752-709.9544$ \\
McD 2.1-m & $710.9034-710.9525$ \\
McD 2.1-m & $711.7642-711.9420$ \\
\hline
\end{tabular}

$0.5 \mathrm{~m}$ telescope used in their observations. Although preliminary observations of LSS 5121 at the Jacobus Kapteyn Telescope (JKT) in 1999 June showed probable variability, LSS 5121 was not the primary target for the observing run, so the observations did not cover enough time for the period search to be conclusive (Aznar Cuadrado et al. 2000). The observations we now report were carried out in 2000 May and June to provide better measurements of the star's variability.

\section{Observations and data reduction}

\subsection{JKT photometry}

Photometric measurements of LSS 5121 were performed using the JAG-CCD camera on the 1-m JKT at the Roque de los Muchachos Observatory, La Palma on the nights of 2000 May 13 and 14. Observations were made using the Cousins $V$ filter. Pixels on the $2048 \times 2048$ chip covered 0.33 arcsec per pixel, giving a usable field of about 10 arcmin. Each night the observations covered about 4.2 hours, with exposures being taken every 3 or $4 \mathrm{~min}$.

\subsection{McDonald photometry}

LSS 5121 was observed on the nights of 2000 June 10, $11,13,14,15$, and 21 using the Prime Focus Corrector of the McDonald Observatory 0.8-m telescope. Observations were made using the Bessel $V$ filter. The camera gives 1.3 arcsec per pixel using the $2048 \times 2048$ pixel CCD, giving a 46 arcmin field. As LSS 5121 is in a fairly crowded field, we were able to reduce the portion of the chip read out, thus reducing the readout time and disk space requirements, while still including enough comparison stars in the observation. The photometric data from both McDonald and JKT were obtained for use as differential photometry. The times covered by observations at JKT and McDonald are listed in Table 1.

\subsection{McDonald spectroscopy}

Spectra of LSS 5121 were obtained on the nights of 2000 June 13, 14, and 15 using the Sandiford Echelle spectrograph (McCarthy et al. 1993) on the 2.1-m Otto Struve

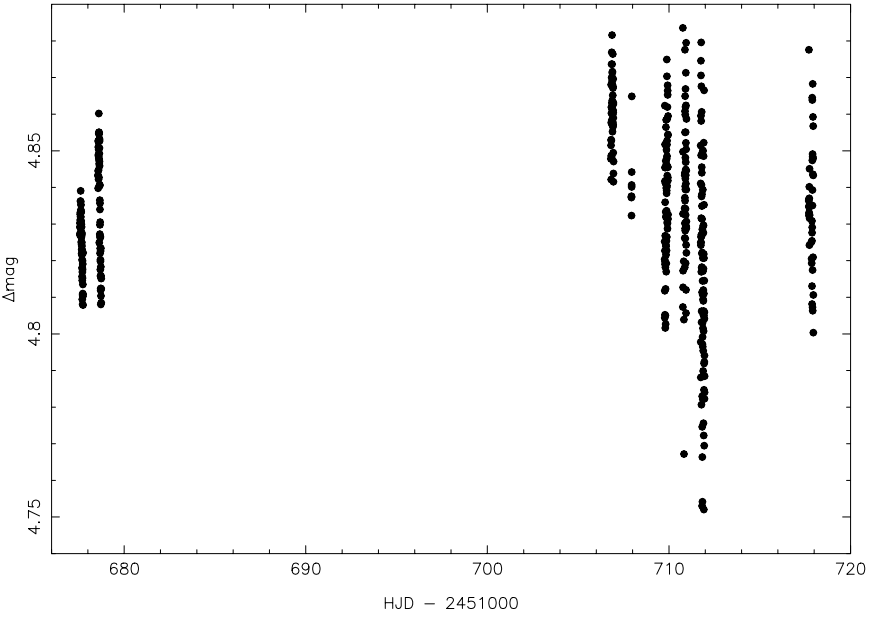

Fig. 1. Differential photometry from the JKT and McDonald runs described in Table 1

Telescope. Repeated 15 to 20 min exposures were made during the times listed in Table 1 to allow us to detect changes in the spectrum due to pulsation. Spectra typically had a signal to noise ratio between 7 and 11 . The wavelength range of the echelle orders overlapped, so the spectra give continuous coverage between 4410 and $4960 \AA$. We used a 1.65 arcsec slit width, which gives a spectral resolution of $\lambda / \Delta \lambda \approx 40000$.

\subsection{Data reduction}

Photometric data were reduced using standard IRAF packages to subtract bias and divide by flats. IRAF's PHOT package was used to do aperture photometry on the images. Stars in uncrowded parts of the field and falling on parts of the CCD without bad pixels or columns were chosen for use as comparison stars for the differential photometry by visually inspecting the images.

Spectra were reduced using standard IRAF packages for bias and flat field correction, reducing echelle orders to one dimensional spectra, and applying the wavelength scale using thorium-argon arc spectra. Velocity corrections for Earth's motion were found for each exposure using RVCORRECT and were applied using DOPCOR.

\section{Analysis}

\subsection{Differential photometry}

Comparison stars were chosen for each night's data. To be chosen, a star needed to be present on a good part of the chip for every LSS 5121 image for the night. The flux measured for LSS 5121 and the combined flux measured for the comparison stars were used to find $\Delta \mathrm{mag}=m_{V}(\mathrm{LSS} 5121)-m_{V}($ comparison $)$. Corrections were then made to put all the data on the same scale, taking into account that different reference stars were used on separate nights. Differential photometry for the two observing runs are shown in Fig. 1. 


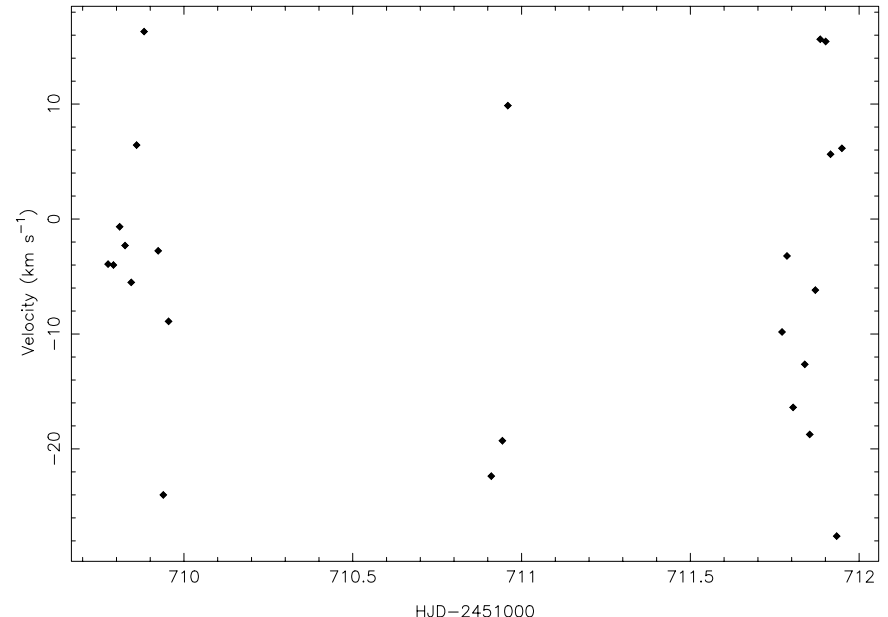

Fig. 2. Radial velocities measured for LSS 5121

\subsection{Radial velocities}

Velocity shifts were measured using the cross correlation package FXCOR in IRAF. The spectra were coadded to provide a template with a signal to noise ratio of about 35 . Only the two echelle orders covering 4460 to $4500 \AA$ and 4880 to $4930 \AA$ had features, notably the $4471.5,4920.8$ and $4921.9 \AA$ He I lines, strong enough for the cross correlation to yield a velocity. We gave more weight to the velocities found using the longer wavelength order, as the order containing the $4471.5 \AA$ line had lower signal. Other orders contained lines that may be useful for velocity determination in studies using larger telescopes, but which did not give reliable cross correlation peaks with our spectra. The velocities thus found were applied and the resulting spectra were coadded to give a template with smaller velocity smearing for a second iteration. Velocities found for three nights are displayed in Fig. 2. We estimate uncertainties in radial velocity determinations to be about $\pm 10 \mathrm{~km} \mathrm{~s}^{-1}$. This is a rough estimate based on how accurately we could determine the center of the cross correlation peak.

\section{Results}

The uncertainties for the radial velocity data are large compared to differences between the calculated velocities: we cannot say that radial velocity variations are obvious. The differential photometry shows evidence that LSS 5121 varies on a timescale of a few hours, however no period was immediately apparent. The photometry measured at JKT shows a magnitude change of about 0.04 mag over a period of about 2.5 hours. The photometry measured the next month at McDonald shows similar variation, though the data are noisier. Uncertainties for the photometric data varied with the weather, but in general we estimate uncertainties of $\pm 0.003 \mathrm{mag}$ for the JKT data and $\pm 0.015 \mathrm{mag}$ for the McD data. Noise was reduced by finding the mean for the photometric data in 0.02 day bins (Figs. 3 and 4). Tests with non-variable comparison stars with magnitudes

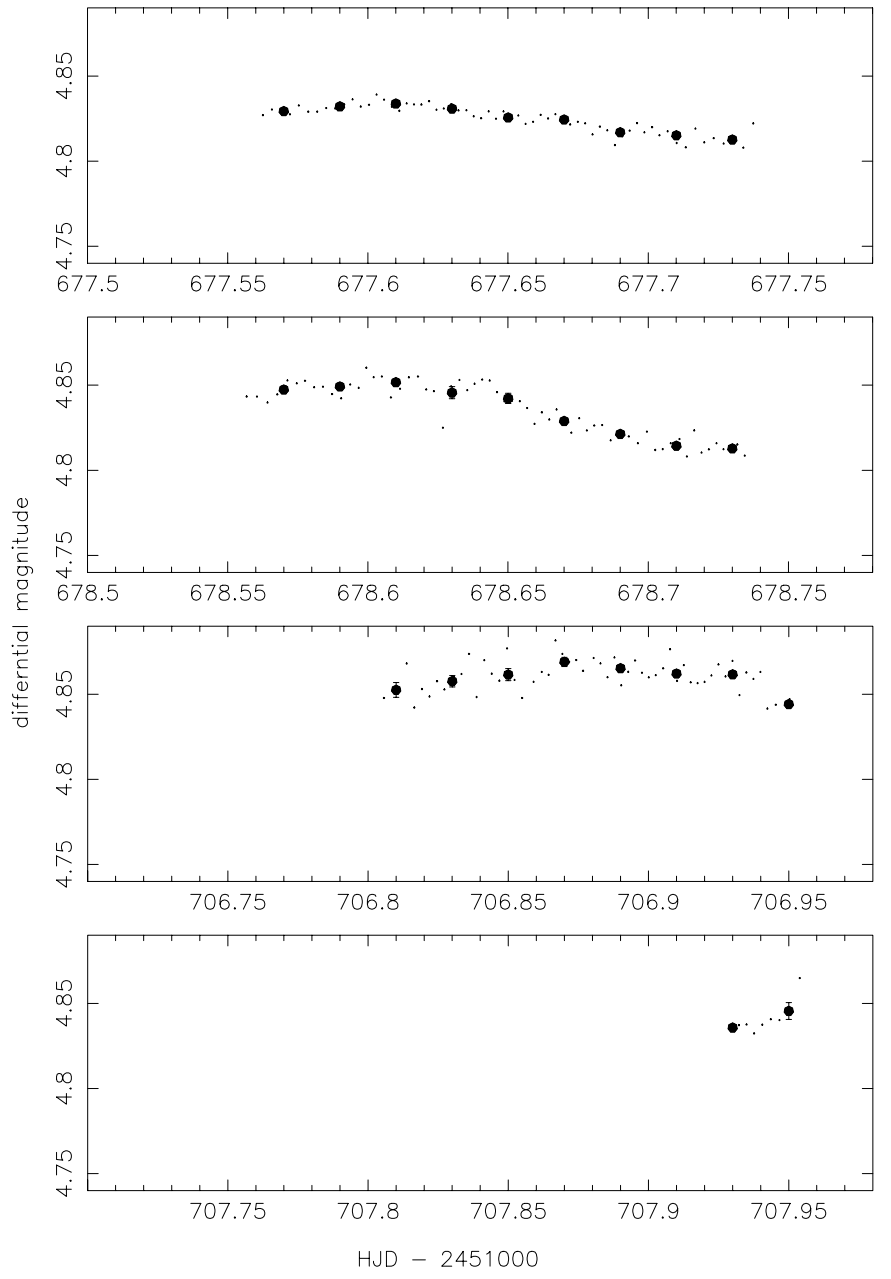

Fig. 3. Differential photometry for LSS 5121. Small points are measured photometry values. Large points indicate average photometry in 0.02 day bins, with error bars showing standard deviation of the mean in each bin. Top two panels are JKT data. Bottom two are McDonald data. Error bars are smaller than the large data points for the JKT data

similar to LSS 5121 gave differential photometry which did not vary more than about $0.01 \mathrm{mag}$.

Periodograms and window functions were found for the photometric and velocity data using DIPSO's PDGRAM and PDGWIN features (Scargle 1982). The velocity periodogram has no major peaks, which is not surprising, given the small number of data points. The photometry periodogram has no major peaks that are not also in the data's window function (Fig. 5). The major peaks in both are near multiples of the one day alias. Data were phased to the periods indicated by minor peaks in the periodogram not present in the window function, but this provided no further evidence of periodic behavior. We cannot rule out that LSS 5121 varies with a period of about 1 day or that it varies with longer periods and/or smaller amplitudes than our observations could detect. 

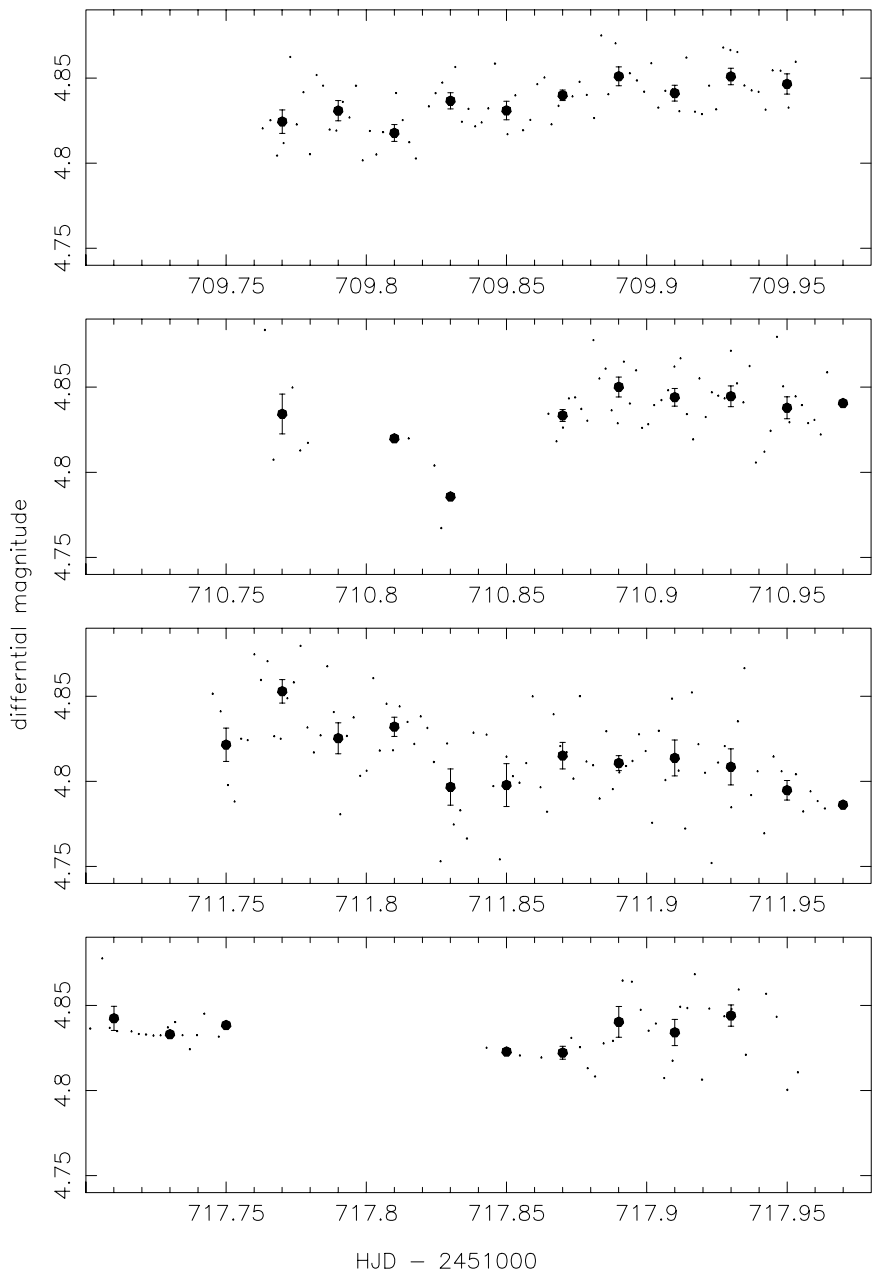

Fig. 4. McDonald differential photometry. Symbols as in Fig. 3

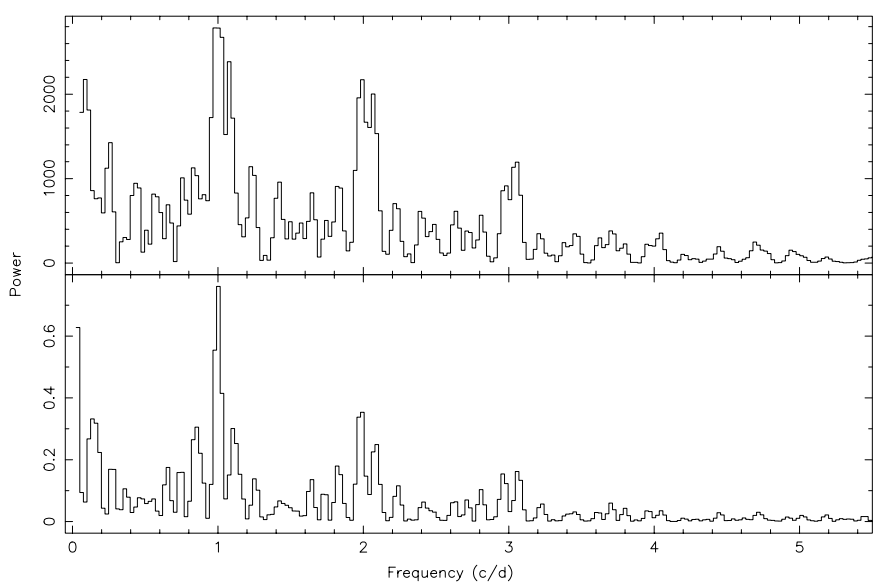

Fig. 5. Periodogram (top panel) and window function (bottom panel) for photometric data

\section{Discussion and conclusions}

Differential photometry shows that LSS 5121 varies in brightness, with changes of up to 0.04 mag over a span of 2.5 hours. LSS 5121 was previously known to have varying spectral lines. We have now shown that it varies in brightness as well. Analysis of data from observations on

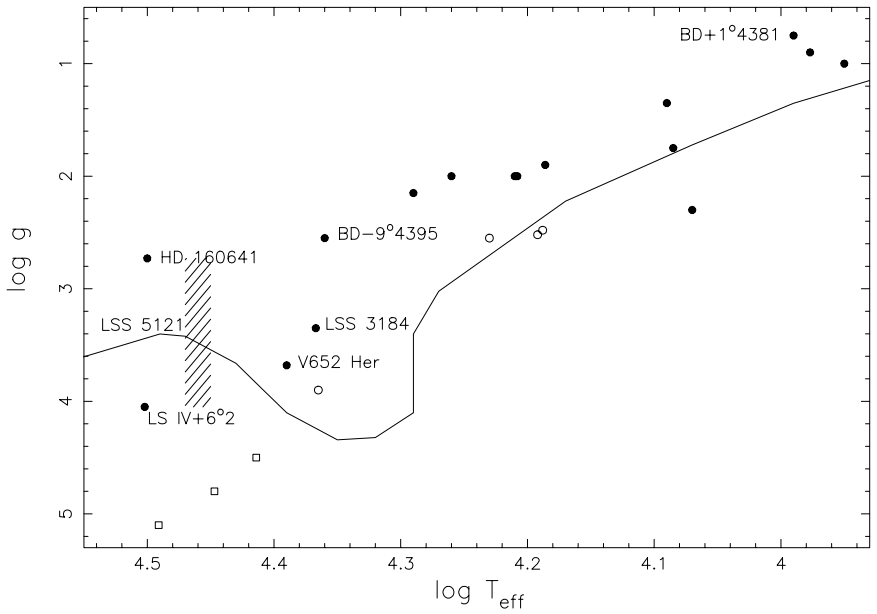

Fig. 6. Instability boundary (solid line) in the $\log g-\log T_{\text {eff }}$ plane for $0.7 M_{\odot}$ helium stars with $X=0.00$ and $Z=0.01$. EHes are marked with circles. The properties of the EHe LSS 5121 fall in the hatched area. Three He sdB stars are shown by squares. Stars known to vary are indicated by filled symbols

8 nights in 2000 May and June did not yield evidence of any periodicity in the variations. Our search for variability in radial velocity was inconclusive. A successful radial velocity search will require a larger telescope to provide higher signal to noise spectra.

Saio (1995) showed that the region of the HR diagram where pulsations should occur overlaps part of the region where EHes appear. Figure 6 shows the location of all known EHes with known $T_{\text {eff }}$ and $\log g$, and the Fe-group opacity instability boundary for $0.7 M_{\odot}$ helium stars in the $\log g-\log T_{\text {eff }}$ plane. The EHes mentioned in the text are labeled in the figure. Helium stars above the boundary should be unstable against pulsation. The exact location of the instability boundary depends on stellar mass and chemical composition.

In general, the observed pulsation timescales in EHes decrease with higher temperature (Saio \& Jeffery 1988). Two EHes, LSS 3184 (BX Cir) and V652 Her pulsate in their fundamental radial modes with well determined periods of about 2.6 hours (Kilkenny et al. 1996, 1999). Pulsations in the coolest EHes (e.g. BD $+1^{\circ} 4381$ ) seem to be non-periodic or chaotic with variation timescales of 8 to 25 days (Lawson et al. 1993). Other EHes with known variability seem to pulsate non-radially, some with multiple periods. For example, Lynas-Gray et al. (1987) report finding periods of $0.35,0.71,1.12$, and 1.77 days in the photometry of HD 160641, an EHe with a temperature similar to that of LSS 5121 and Jeffery et al. (1985) report 3.5 day, 11.2 day, and possibly additional periods for BD- $9^{\circ} 4395$. It is possible that chaotic pulsations also affect some of the hotter EHes, so that some of the multiple periods found for the hot non-radial pulsators would more accurately be referred to as variability timescales or "quasi-periods", and that different periods or no periods 
would be found if data from longer, more continuous observations were available.

Any pulsations of LSS 5121 are either non-periodic or have periods which our data cannot resolve. Our inability to find a period is consistent with LSS 5121 being a nonradial or a chaotic pulsator, though it does not constitute proof. Its pulsations are probably similar to those of the hot extreme helium stars BD-9 ${ }^{\circ} 4395$ and HD 160641, both of which have been reported to pulsate non-radially with multiple periods (Jeffery \& Heber 1992; Lynas-Gray et al. 1987).

Acknowledgements. We acknowledge financial support from the UK PPARC (grant Refs. PPA/G/S/1998/00019 and PPA/G/O/1999/00058). VW thanks Marcel Bergmann for instruction on use of the 0.8-m telescope and Nairn Baliber for a refresher on photometry reduction. GP received support from grant AST 9618414 from the NSF.

\section{References}

Aznar Cuadrado, R., Montañés Rodrígues, P., \& Jeffery, C. S. 2000, in The Impact of Large-Scale Surveys on Pulsating Star Research, ed. L. Szabados, \& D. Kurtz, ASP Conf. Ser., 203, 438

Heber, U., Jonas, G., \& Drilling, J. S. 1986, in Hydrogen Deficient Stars and Related objects, ed. K. Hunger,
D. Schönberner, \& N. K., Rao, IAU Coll., 87, 69

Jeffery, C. S., \& Heber, U. 1992, A\&A, 260, 133

Jeffery, C. S., Heber, U., \& Hill, P. W., et al. 1996, Hydrogen Deficient Stars, ed. C. S. Jeffery, \& U. Hebe, ASP Conf. Ser., 96, 471

Jeffery, C. S., Skillen, I., Hill, P. W., et al. 1985, MNRAS, 217, 701

Jeffery, C. S., Starling, R. L. C., Hill, P. W., et al. 2001, MNRAS, in press

Kilkenny, D., Koen, C., Jeffery, C. S., et al. 1999, MNRAS, 310,1119

Kilkenny, D., Lynas-Gray, A. E., \& Roberts, G. 1996, MNRAS, 283, 1349

Lawson, W. A., \& Kilkenny, D. 1998, Observatory, 118, 1

Lawson, W. A., Kilkenny, D., van Wyk, F., et al. 1993, MNRAS, 265, 351

Lynas-Gray, A. E., Schönberner, D., Hill, P. W., et al. 1984, MNRAS, 209, 387

Lynas-Gray, A. E., Kilkenny, D., Skillen, I., et al. 1987, MNRAS, 227, 1073

McCarthy, J. K., Sandiford, B. A., Boyd, D., et al. 1993, PASP, 105,881

Saio, H. 1993, MNRAS, 260, 465

Saio, H. 1995, MNRAS, 277, 1393

Saio, H., \& Jeffery, C. S. 1988, ApJ, 328, 714

Saio, H., \& Jeffery, C. S. 1999, MNRAS, 208, 221

Scargle, J. D. 1982, ApJ, 263, 835

Woolf, V. M., \& Jeffery, C. S. 2000, A\&A, 358, 1001 